\title{
SOLUCIÓN DE COMERCIO ELECTRÓNICO PARA LA COMERCIALIZACIÓN DEL PRODUCTO “TU EXCURSIÓN CONMIGO” EN LA AGENCIA DE VIAJES CUBANACÁN SUCURSAL VILLA CLARA.
}

\author{
Maité Rodríguez González \\ Universidad Central “Marta Abreu” de Las Villas. \\ Cuba \\ maiterodriguez@uclv.edu.cu \\ Adrian González Oliva \\ Universidad Central "Marta Abreu” de Las Villas. \\ Cuba \\ adrianoo@uclv.edu.cu
}

\section{RESUMEN}

Tu Excursión Conmigo (TEC) es una de las más recientes ofertas de las Agencias de Viajes en Cuba. Específicamente, la gerencia de la Agencia de Viaje Cubanacán Villa Clara se encuentra enfocada en la mejora continua de las TEC para aumentar los niveles de satisfacción de los clientes. Esto sobrelleva complejos procesos decisionales que consumen grandes cantidades de información. Las principales limitaciones que tiene la gerencia en este contexto son la incapacidad de los sistemas de gestión vigentes de capturar y reflejar información relevante de las TEC, debido a que están orientado únicamente a asistir la gestión contable y financiera, y no contar con sistemas que brinden información requerida en el momento oportuno para la asistencia a las decisiones operativas, tácticas y estratégica que son neurálgicas para el logro de sus objetivos. Las Tecnologías de la Información y las Comunicaciones (TIC) desplazaron la llamada "Era industrial" para dar paso a la joven "Era de la información", con reconocidos aportes en el área de la asistencia decisional y marcado incremento de las aplicaciones en la gestión turística. En esta investigación se presenta una solución de comercio electrónico para la comercialización de las TEC en la Agencia de Viajes Cubanacán Villa Clara.

Palabras claves: Comercio electrónico, Agencia de Viajes, Gestión. 


\title{
AN E-COMMERCE SOLUTION FOR “YOUR TRIP WITH ME” PRODUCT MANAGEMENT AT THE CUBANACÁN TRAVEL AGENCY, VILLA CLARA BRANCH, CUBA
}

\author{
Maité Rodriguez González \\ Central University “Marta Abreu” of Las Villas. \\ Cuba \\ maiterodriguez@uclv.edu.cu \\ Adrian González Oliva \\ Central University "Marta Abreu” of Las Villas. \\ Cuba \\ adrianoo@uclv.edu.cu
}

\section{ABSTRACT}

Your Trip with Me (YTWM) is one of the latest offers from travel agencies in Cuba. Specifically, the management of the Travel Agency Cubanacán Villa Clara is focused on continuous improvement of YTWM in order to increase levels of customer satisfaction. This undertakes complex decision-making processes that consume large amounts of information. The main limitations of the management in this context are the existing management systems inability to capture and reflect relevant information from the YTWM, because they are only oriented to assist the accounting and financial management, without having systems to provide the required information in a timely manner to assist operational, tactical and strategic decissions are key to accomplish the objectives of the entity. The Information Technology and Communications (ICT) replaced the "industrial age" to make way for the young "Information Age" with recognized contributions in the area of the decision-making and marked increase applications in tourism management. This research presents an e-commerce solution for the marketing of the TEC in the Travel Agency Cubanacán Villa Clara.

Key words: e-commerce, Travel Agency, Management. 


\section{INTRODUCCIÓN}

Las TEC son excursiones de reciente creación en Cuba, como alternativa de desarrollo de la economía y para incentivar e incrementar las prácticas recreativas de los habitantes de la isla. Su surgimiento ha tenido un impacto directo sobre la economía, la política y la sociedad cubana. Sugiere un cambio de paradigma tanto para la concepción del turismo nacional como para la gestión de las Agencias de Viajes (AAVV), principalmente las agencias receptivas. La Agencia de Viaje Cubanacán Villa Clara hereda directamente las consecuencias de este suceso, es una Agencia de Viajes clasificadas como "receptoras" y que comienza a comportarse como "emisora" al mercado nacional.

Las TEC en la agencia objeto de estudio, marcan sin dudas la necesidad de replantear el modelo de gestión para adaptarse de manera ágil al nuevo contexto. Podría resultar de utilidad introducir y capitalizar las ventajas de las Tecnologías de la Información y las Comunicaciones (TIC) en la comercialización y distribución de las TEC, lo que se conceptualiza como comercio y marketing electrónico.

Por ello la presente investigación tiene como objetivo general realizar una propuesta de mejora o rediseño del proceso de distribución del producto TEC, a partir del análisis de su situación actual enfocada al uso de soluciones de comercio electrónico.

\section{Antecedentes}

Investigaciones realizadas a la Agencia de Viajes Cubanacán Villa Clara durante años anteriores, específicamente 2009, 2010 y 2011, así como los resultados económicos obtenidos en la venta del producto TEC, testifican la gran aceptación por parte de los ciudadanos cubanos, principalmente, con respecto a dichas opcionales turísticas, pues ha pesar de existir algunos problemas de gestión en sus inicios, se han encontrado un por ciento elevado de clientes, nacionales y extranjeros, que han acudido a esta y demás Agencias de Viajes para efectuar la reservación y hacer uso de estos servicios.

Sin embargo, es notorio aclarar que al insertarse la venta de esta opcional en la agencia, esta no contaba con un desglose que delimitara los resultados obtenidos por concepto de las nuevas excursiones económicas implementadas en el país. No es hasta el año 2010 que se han introducido los datos de manera separada en las cuentas financieras y económicas de la agencia. Sin embargo aún es insuficiente estos cambios efectuados en la gestión del producto TEC, con ello solo se lograría que la entidad realizara algunos análisis económicos-financieros para investigar el comportamiento del producto en un período determinado.

Por tanto, si la agencia aspira a realizar análisis relacionados con la toma de 
decisiones en beneficio de la entidad y de los clientes, este sistema resulta ineficaz para insertar a la agencia en las nuevas tendencias empresariales referidas a la implementación de novedosas herramientas gerenciales y los sistemas basados en las Nuevas Tecnologías de la Información y las Comunicaciones (TIC). La agencia carece de estos instrumentos, los cuales pueden ayudar a desarrollar el E-commerce y E-marketing en la gestión de la agencia y, en específico, del producto TEC; no cuenta con la tecnología idónea para insertarse y adaptarse a las nuevas tendencias gerenciales relacionadas con las TIC.

\section{Metodología}

En el desarrollo de la investigación se utilizaron tanto métodos cuantitativos como cualitativos. Dentro de los primeros se utilizaron los métodos estadísticos, mientras que las técnicas de trabajo en grupo, las encuestas, la observación, las entrevistas, a los métodos cualitativos.

Se realizaron además estudios de programación lineal y de inteligencia artificial. La propuesta de mejora o rediseño del producto TEC está basada en una plataforma de integración de la información basada en tecnología Web. Se desarrolló utilizando como sistema de gestión de bases de datos Microsoft SqlServer ${ }^{\circledR}$ para el almacenamiento de la información; Apache, para el servidor de servicios Web y PHP como lenguaje de programación para la captura, procesamiento y distribución de la información. Para lograr las funcionalidades de descubrimiento de reglas de asociación y segmentación del mercado se sugiere el uso de la librería de Weka ${ }^{\circledR}$. Las tecnologías en las que se apoya para la recuperación y procesamiento de la información son: 1-Lenguajes estructurado de consultas (SQL), 2- Microsoft Excel® ${ }^{\circledR}$ 3- SPSS17®

Se realizó un análisis de sensibilidad y segmentación del mercado utilizando el método RFM (Recency, Frecuently, Money), lo que permite determinar comportamientos y tendencias en el consumo de Tu excursión conmigo.

\section{RESULTADOS}

\section{Situación actual del proceso de comercialización del producto TEC.}

Teniendo en cuenta además que las teorías referentes a la mejora continua y rediseños de procesos apuntan, como etapa básica, al análisis del proceso actual, resulta de relevante importancia para la investigación analizar el proceso de comercialización del producto TEC en la Agencia de Viajes Cubanacán, el cual se explica brevemente a continuación:

El proceso inicia con la recepción (ver Figura 1) de las relaciones de precios de 
las entidades hoteleras del país, que envía la casa matriz a través de correo electrónico. Se gestiona de manera sincrónica las relaciones de precio de los transportistas locales. La información de hospedaje y transporte se valida de errores estructurales que puedan comprometer la calidad del resto del proceso.

Posterior a ello se diseñan las excursiones, donde se basan principalmente en la experticia de los encargados del diseño e informaciones de naturaleza económica que maximizan los beneficios de la Agencia de Viajes en consenso con las características económicas del mercado nacional.

La venta, se realiza en el Buró de Venta de la Agencia de Viajes y en los hoteles ubicados en los Cayos del Norte de la provincia. El cliente se dirige a estas intermediarias para solicitar información y asesoría con respecto a las nuevas ofertas turísticas. La promotora de venta se encarga de brindar una explicación detallada de cada producto turístico por el cual esté interesado el cliente, apoyándose la mayoría de las ocasiones en la carpeta destinada a ofrecer información sobre el producto TEC. Esta contiene imágenes y los servicios que incluye el producto en cuestión.

Figura $\mathrm{N}^{\circ} 1$. Proceso de comercialización de la TEC.

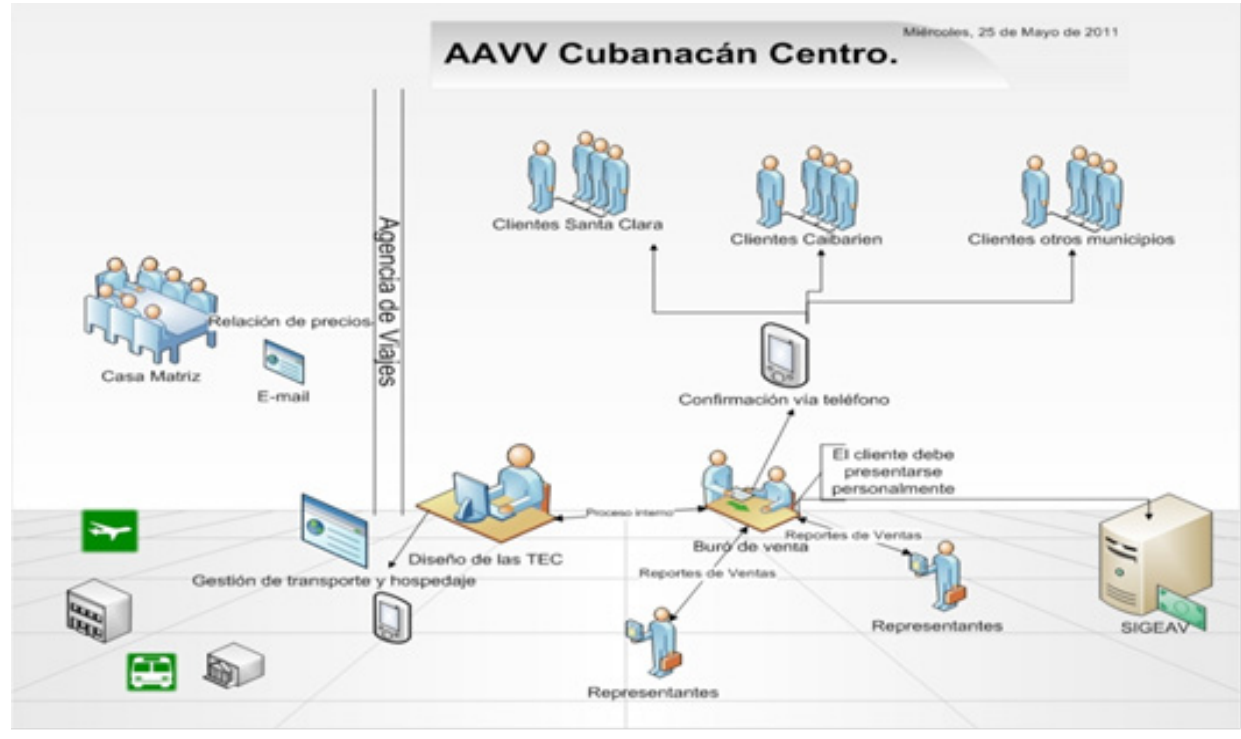

Fuente: Elaboración propia. 
La promotora, cuando el cliente se ha decidido a comprar determinada TEC, procede a efectuar la venta del mismo. Le solicita al cliente, que efectuará la compra, nombre, cantidad de adultos y niños y nombre del hotel. Todo ello, en conjunto con la fecha y el precio, son los datos que incluye durante el llenado del ticket de venta. Inmediatamente la promotora le pide un número de teléfono que le permita comunicarse con el cliente para efectuar la confirmación de la opcional.

Una vez que la promotora ha realizado todas las coordinaciones pertinentes con el mínimo de paxs que exige cada excursión, realiza por vía telefónica, la confirmación de las opcionales. Los datos obtenidos de la venta de cada opcional son registrados en el SIGEAV, sistema de gestión utilizado por la Agencia de Viajes Cubanacán.

Partiendo de la caracterización del proceso de las TEC es posible identificar varias limitaciones desde el enfoque de competitividad empresarial y las cuales se pueden entender como debilidades en los enfoques de gestión de la Agencia de Viajes Cubanacán Villa Clara:

1. La gestión de las relaciones de precios de las transportistas es asincrónica.

2. El sistema existente no brinda información suficiente para realizar segmentos del mercado basadas en características no económicas; realizar análisis de tendencias y comportamiento de los clientes.

3. Lo anterior enunciado es la causa de la no existencia de un enfoque al cliente efectivo.

4. Para obtener información y reservar el cliente debe acudir personalmente. Puede considerarse una debilidad crítica debido a las distancias que se encuentran los municipios de la capital provincial.

5. Las estrategias de publicidad y promoción de las TEC no logran atraer ni fidelizar a los clientes.

\section{Propuesta de solución de comercio electrónico para la comercialización de las TEC}

Las Tecnologías de la Información y las Comunicaciones (TIC) y los modelos de E-commerce pudieran brindar soluciones que permitan transformar las debilidades en fortalezas. No pocas son las ventajas, si se analizan las buenas prácticas de distintas entidades, que se apoyan en el comercio electrónico. Estas sugieren que se experimenta:

1. Mejora en los procesos.

2. Reducción en la necesidad de capital.

3. Nuevos clientes.

4. Mayores ingresos de clientes actuales.

5. Desarrollo de nuevos productos y servicios.

6. Refuerzo de marca. 
7. Fidelización de los clientes.

8. Mejora de la satisfacción de los clientes.

Como posible solución a las limitaciones del sistema actual de la Agencia de Viajes Cubanacán Villa Clara, se presenta una propuesta de una plataforma de integración de la información basada en tecnología Web, y la cual asiste a las funciones de las dimensiones: cliente, prestatarios y entidad.

Los clientes potenciales se encuentran ubicados en los distintos municipios de la provincia Villa Clara, distantes en algunos casos a más de $100 \mathrm{Km}$ de la capital provincial. En la situación actual deben trasladarse hasta la agencia para adquirir las TEC u obtener información, que en este segundo caso puede hacer uso de la telefonía, pero mil palabras no es una imagen.

En la página Web los clientes podrán acceder a las diversas excursiones, con información que contenga altos niveles de detalles cualitativos y cuantitativos, que le permitan tomar la decisión óptima según sus expectativas y poder adquisitivo. La compra podrá realizarla por este medio realizando transferencias monetarias a la cuenta bancaria de la agencia.

La URL de la página debe definirse por la AAVV teniendo en consideración que debe posicionarse en las mentes de los clientes.

En el país cubano existen tendencias conservadoras a criticar las soluciones que requieren medios de cómputos y conexión a Internet basados en las escasas existencias de estos recursos en el hogar de la familia cubana. Sin embargo en los últimos años se ha experimentado un incremento del acceso a Internet de los cubanos utilizando los servicios de las universidades, centros de trabajos y Joven Club de Computación. 
Figura $\mathrm{N}^{\circ}$ 2. Solución de comercio electrónico para la comercialización de las TEC.

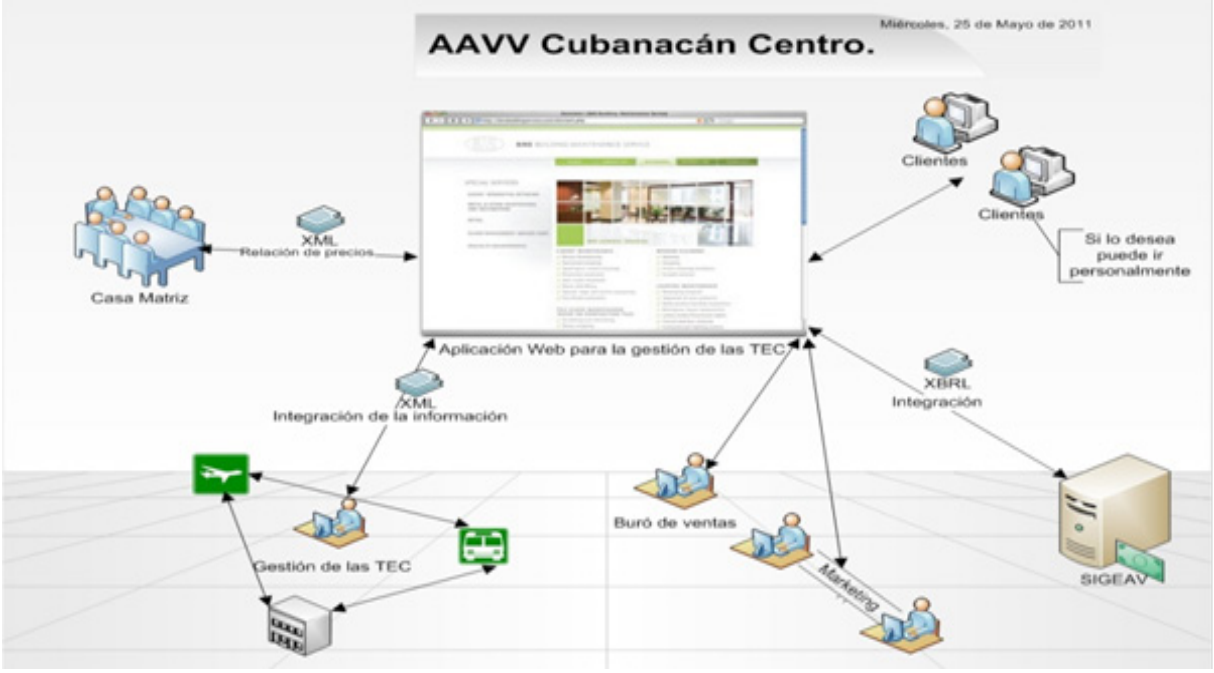

Fuente: Elaboración propia.

Los distintos prestatarios contarán con la posibilidad de publicar la información de sus servicios, incluyendo disponibilidad y precios. El plan de disponibilidad debe ser actualizado en tiempo real para una mejor gestión integral. Además presentan los mecanismos necesarios de comunicación (E-mail, Chat y contactos) entre los prestatarios y la agencia. La información publicada estará disponible sólo para la Agencia de Viajes. La transferencia de información inter-entidades se realizará utilizando el formato XML (eXtensible Marckup Language, por sus siglas en inglés). Este formato es tan conocido como utilizado en las soluciones de integración de información, surgió por la necesidad de reducir la heterogeneidad estructural y sintáctica que presupone los adelantos tecnológicos y altos volúmenes de nuevas soluciones en Internet, donde el comercio electrónico es un ejemplo de uso en el intercambio de información electrónica.

La Agencia de Viajes en la solución propuesta tiene dos dimensiones de funcionalidad, debido a que explotará los servicios de la plataforma de integración para los procesos internos, creación de estrategias y para la publicidad y promoción de las TEC. para:

Como parte de los procesos internos la Agencia de Viajes accederá a la plataforma

1. Intercambio de información entre la Agencia de Viajes y la Casa Matriz.

2. Obtener la información de los servicios de los prestatarios para la creación de las 
TEC.

3. Abrir canales de comunicación tanto con los prestatarios como con los clientes.

4. Integrar la información contable al SIGEAV.

5. Acceder a la información del comportamiento de los clientes para realizar la segmentación del mercado utilizando el método RFM (Recency, Frecuntly, Money; por sus siglas en inglés) y por el método de K-means.

6. Aplicar minería de datos para descubrir reglas de asociación entre los clientes.

7. Diseño de nuevos productos.

También podrá definir las estrategias de E-marketing, principalmente en la publicidad y promoción de las TEC, específicamente estará en condiciones de:

1. Publicar las TEC que se ofrecen en la Agencia.

2. Servicios de infomediación.

3. Enviar E-mail a los clientes con confirmaciones y ofertas de interés.

4. Uso de Banners para resaltar ofertas especiales y descuentos.

5. Encuestas on-line de satisfacción del cliente.

6. Emisión de boletines electrónicos.

7. Servicio de búsqueda rápidas.

8. Atención en línea mediante el uso del Chat.

Los temas relacionados con la seguridad y legalidad queda fuera del alcance de la investigación. Debe considerarse que son considerados "maduros" en los modelos de E-commerce existentes.

Como características generales adicionales de la plataforma Web, se incluyen otras especificaciones resultados de las buenas prácticas en el sector, tales como:

1. Logotipo.

2. Posibilidad de elegir el idioma.

3. Enlace o sección de Preguntas Frecuentes (Frecuently Asked Question, por sus siglas en inglés).

4. Restricciones legales o especiales de comportamiento.

5. Formulario de inscripción en línea.

6. Señala los plazos de cancelación o devolución parcial y los reembolsos posibles.

7. Informa sobre el nivel de seguridad de la transacción y los certificados del sitio.

\section{Implementación del sistema de gestión integral}

El sistema se implementó utilizando tecnologías Web, sigue el patrón MVC (Model-View-Controler).Se siguió una estrategia de metodología hábil y el lenguaje de 
modelado unificado.

Figura № 3. Diagrama de secuencia de información para la reservas de las TEC.

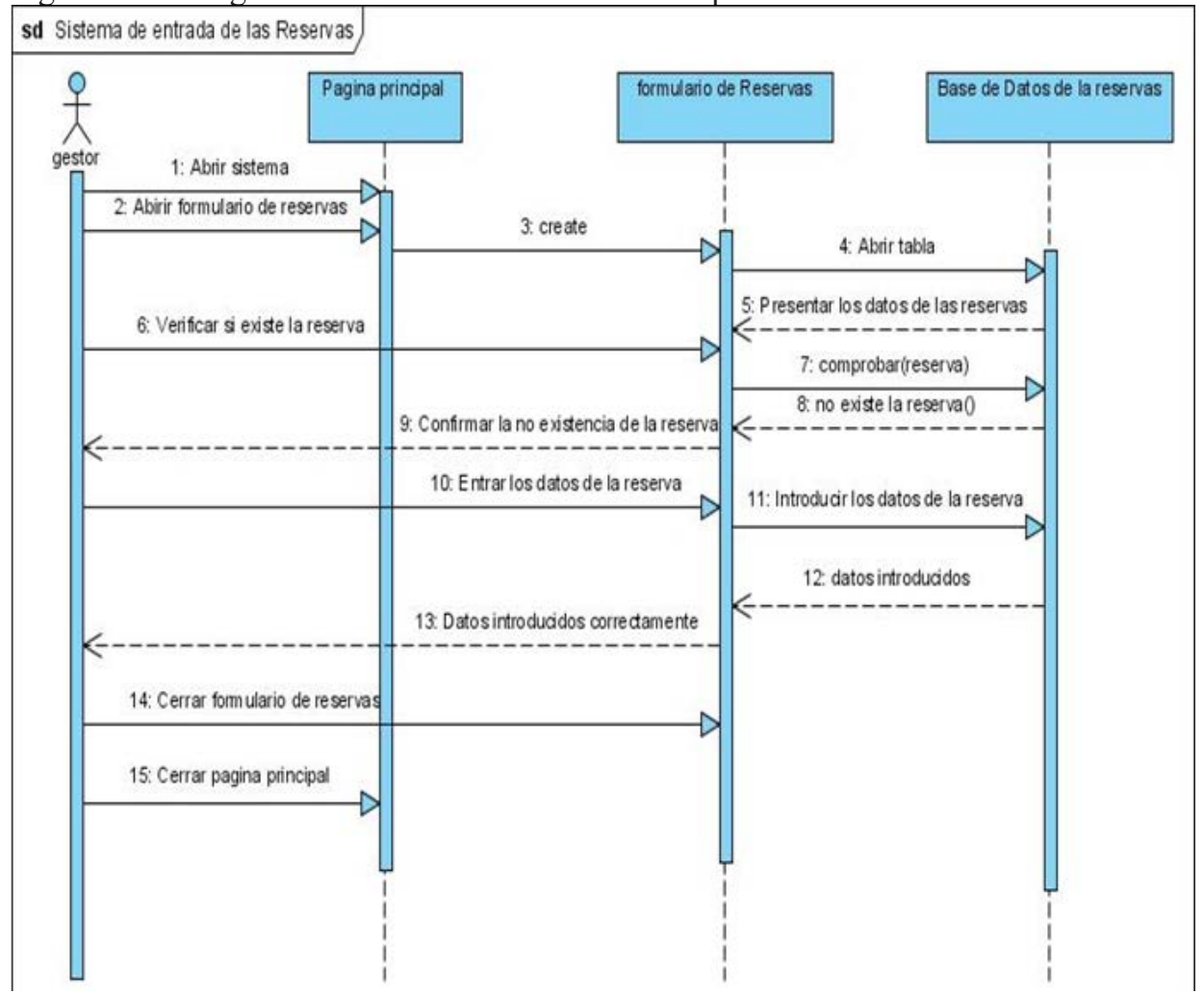

Fuente: Elaboración propia.

Un primer caso de uso corresponde a los procesos de reserva y contratación, los responsables de las actividades entrarán al sistema y podrá acceder a las opciones de reservas, gestión de transporte y gestión de hoteles.

Un segundo caso de uso es el del administrador del sistema, encargado de la gestión de seguridad, configuración y mantenimiento. La actividad principal de este actor es garantizar que los listados del proceso de investigación de mercado, diseño de nuevos productos y listados de precios enviados por la Casa Matriz estén actualizados.

EI tercer actor representa a los encargados de la gerencia que utilizarán el sistema para integrar la información con el sistema de gestión de la Agencia de Viajes 
(SIGEAV), integración de la información necesaria porque el SIGEAV se orienta solo a la gestión contable. Además el sistema les proporcionará los reportes necesarios para el proceso de toma de decisiones orientado a estudios de mercado, perfiles de clientes e indicadores económicos financieros.

El sistema de gestión integral brinda la posibilidad de realizar reservas, realizar la gestión de transporte, gestión de hoteles, la gestión de los procesos internos, información variada de contactos de la entidad y suministradores de servicios, herramientas de comunicación como chat y correo electrónico y los medios para la gestión de marketing electrónico.

Un ejemplo concreto es el de la gestión de reservas que puede accederse utilizando la barra de menú horizontal o vertical:

En la página de reserva se podrá ingresar una nueva reserva, revisar la lista de reserva existente, modificar las propiedades de la reserva, confirmar las reservas a clientes y suministradores de servicios vía correo electrónico.

Figura № 4. Sistema de gestión de reservas de las TEC.

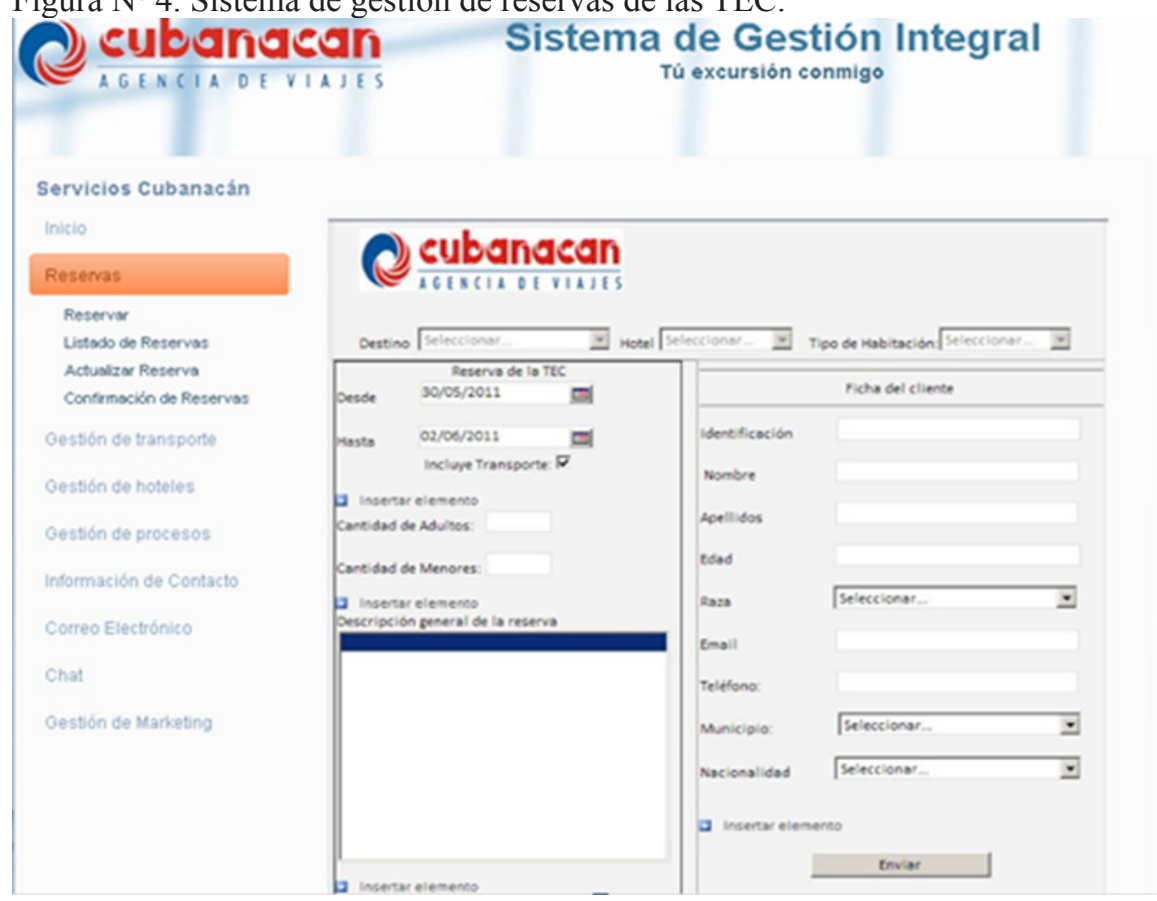

Fuente: Elaboración propia. 


\section{CONCLUSIÓN}

El enfoque actual de la Agencia de Viajes Cubanacán Villa Clara no está claramente orientado al cliente pues los sistemas de gestión actuales no brindan la información requerida para lograrlo y no se explota al máximo las TIC para las actividades de comercio y publicidad. Por ello las soluciones de E-commerce constituyen una ventaja competitiva para la Agencia de Viajes objeto de estudio dado que reduce los costos, brinda información más completa del mercado y su comportamiento y ofrece las herramientas necesarias para aumentar la satisfacción del cliente.

\section{BIBLIOGRAFIA}

Braun, P. (2002). Networking Tourism SMEs: e-Commerce and e-Marketing. Information Technology and Tourism. 5(1): 13-23.

H Werthner, F.R. (2004). E-commerce and tourism.

Lab, e.-C.a.T.R. (2002). User modelling and decision making in travel and tourism emergent systems.

Tjoa, A.M. (2004). E-Commerce and Tourism: Retrospective and Perspective.

Wang, Y. (2001). Defining the virtual tourist community: implications for tourism marketing Tourism Management. 23(4): p. 407-417.

Lans, R.F.v.d. (2007). SQL for MySQL developers: a comprehensive tutorial and reference. Addison-Wesley.

Ma, Z. (2007). Intelligent Databases:Technologies and Applications. Idea Group Publishing.

Silberschatz, A. (2002). Fundamentos de bases de datos, ed. C.F. Madrid. España: McGRAW-HILL/INTERAMERICANA DE ESPAÑA, S. A. U.

Whitehorn, M., Marklyn, Bill (2007). Inside Relational Databases with Examples in Access. Springer.

Recibido: $26 / 03 / 2012$

Aceptado: 11/06/2012

Arbitrado anónimamente 